\title{
Heat transfer modeling by integral boundary-layer methods towards icing applications
}

\author{
Rémi Harry*, Emmanuel Radenac ${ }^{\dagger}$, Ghislain Blanchard ${ }^{\ddagger}$ and Philippe Villedieu ${ }^{\S}$ \\ Onera, Toulouse, France, BP 40252 avenue Ed. Belin
}

\begin{abstract}
A 2D modal integral boundary layer code for heat transfers computation was developed in order to allow accurate computation of non-uniform wall temperature flows. The model for this method as well as a previous thermal integral method are described in this paper. The model for both methods is based on solving an integrated form of the energy equation in the boundary layer. An unsteady Finite-Volume formulation is solved until convergence in order to compute the steady solution. This choice allows for an easy extension in three dimensions. Both methods are compared using an in-house code, CLICET, solving directly the Prandtl equations in the boundary layer through local meshing of the boundary layer and a marching algorithm, as a reference solution. Comparison are carried out on auto-similar wedge flows and wing profiles for both uniform and non-uniform imposed wall temperature.
\end{abstract}

\section{Nomenclature}

$u_{e}=$ External velocity

$u_{x}=$ Tangential velocity

$u_{y}=$ Normal velocity

$x=$ Longitudinal abscissa

$y=$ Vertical coordinate, normal to the wall

$\hat{y}=$ Normal distance to the wall normalized by the local thermal boundary layer thickness

$\delta=$ Dynamic boundary layer thickness

$\delta_{1}=$ Displacement thickness

$\theta_{x}=$ Momentum thickness

$C_{f}=$ Friction coefficient

$T=$ Local static temperature

$T_{e}=$ External static temperature

$\phi=$ Normal heat flux

$c_{p}=$ Specific heat capacity at constant pressure

$\tau_{i j}=$ Component $i, j$ of the shear stress tensor

$t=$ Time

$p=$ Static pressure

$\theta_{T}=$ Enthalpy thickness

$C_{D}=$ Dissipation coefficient

$k=$ Thermal conductivity

$\rho \quad=\quad$ Fluid density

$\vec{V} \quad=$ Velocity vector

$S t=$ Stanton number $\phi_{w} /\left(\rho u_{e} c_{p}\left(T_{w}-T_{e}\right)\right)$

\footnotetext{
*PhD Student, Multi-physics department for energy, remi.harry@onera.fr

${ }^{\dagger}$ Research engineer, PhD, Multi-physics department for energy, Emmanuel.Radenac@onera.fr

${ }^{\ddagger}$ Research engineer, PhD, Multi-physics department for energy, Ghislain.Blanchard@onera.fr.

${ }^{\S}$ Research Director, PhD, Multi-physics department for energy, Philippe.Villedieu@onera.fr
} 


\section{Introduction}

Aircraft in-flight icing prediction is one field for which it has remained common practice to calculate convective heat transfer by coupling inviscid fluid and boundary layer codes. Indeed, for both certification and aircraft design, a large number of icing conditions must be calculated within a reasonable time. In addition, the calculation procedure is sequential and quasi-steady. As the ice grows, the shape of the wing or obstacle is updated. The flow needs to be systematically calculated around this updated shape. For a given shape, many aerodynamic calculations may therefore be necessary. As a result, a robust and fast calculation method for convective heat transfer is interesting for in-flight icing applications. This is especially true in 3D because the grids are obviously still larger than in 2D.

As already explained in [1], there are many justifications to the use of integral boundary layers for ice-accretion simulations. In particular, the key expected outputs of the method are the heat exchange coefficient and the skin friction coefficient in the area of the leading edge where the boundary layer is attached. An integral-boundary-layer solver was developed in [1] to solve the dynamic boundary layer. The method was presented in 2D but it was developed with the purpose of an easy enough extension to 3D [2]. It was used successfully for ice-accretion simulations in [3]. The laminar thermal boundary layer was computed in a similar way in [4] under the assumption that the wall temperature is uniform. The issue of non-uniform thermal boundary conditions at the wall is investigated in the present article. The activation of thermal (electro-thermal or bleed-air) ice protection systems indeed leads to non-uniform distributions of wall heat flux and wall temperature in the protected areas.

The aim of the present paper is therefore to present and discuss two different integral methods for the solution of the heat equation in the boundary layer. Attention here is limited to the laminar flow regime which is important for icing applications, as the flow near the leading edge is generally laminar. The case of the turbulent regime will be examined in a future article. Moreover, we restrict our attention to the $2 \mathrm{D}$ case. The focus is put on the model formulation and the ability of different approaches to capture the correct evolution of wall heat flux as well as temperature profiles in the boundary layer. Since the energy equation is a scalar equation, with no specific 3D effects, the extension to 3D is expected to be straightforward.

The paper is divided into 4 parts. The first part is a brief reminder of the dynamic-boundary-layer solution, which is an input to the thermal-boundary-layer model. Two different approaches are then described in the two following sections for the numerical solution of the thermal boundary layer: a single-equation integral model already investigated in [4] and a modal integral model based on the Galerkin method. The two methods are then discussed on several test-cases in the last part of the article.

\section{Dynamic boundary layer computation using an integral method}

Under the assumption that the variation of the temperature-dependent quantities such as the density and the viscosity is negligible over the thickness of the boundary layer, the dynamic and the thermal boundary layers are solved separately. The resolution of the dynamic boundary layer is thus an input to the resolution of the thermal boundary layer. Since it is not the scope of this article, the integral method used here is only briefly exposed. As explained earlier, a 2D approach is investigated. Consequently, the integral method is a 1D time-dependent partial differential equation, where $x$ is the curvilinear abscissa along the investigated object. The system solved is [1, 3]:

$$
\frac{\partial \mathbf{U}}{\partial t}+\frac{\partial \mathbf{F}(x, \mathbf{U})}{\partial x}=\mathbf{S}(x, \mathbf{U})
$$

with:

$$
\begin{aligned}
& \mathbf{U}=\left(\begin{array}{l}
u_{e} \delta_{1} \\
u_{e}^{2} \theta_{x}
\end{array}\right)=\left(\begin{array}{l}
U_{1} \\
U_{2}
\end{array}\right) ; \quad \mathbf{F}=\left(\begin{array}{c}
U_{2} \\
u_{e} U_{2}(f(H)-1)
\end{array}\right) ; \\
& \mathbf{S}=\left(\begin{array}{c}
-U_{1} \frac{\partial u_{e}}{\partial x}+\frac{1}{2} u_{e}^{2} C_{f} \\
\left(u_{e} U_{1}-U_{2}\right) \frac{\partial u_{e}}{\partial x}-\frac{1}{2} u_{e}^{2} C_{f}+2 u_{e}^{3} C_{D}
\end{array}\right)
\end{aligned}
$$

$u_{e}$ is the external velocity, $H$ is the shape factor, $\delta_{1}$ and $\theta_{x}$ are the displacement and momentum thicknesses, respectively. $C_{f}$ is the skin friction coefficient, $C_{D}$ is the dissipation coefficient and $f(H$ is a function described in [1, 3]. For integral methods, closure relations must be established to relate the unknown variables to the solved variables $\mathbf{U}$. Here, in the laminar regime, since all the data are dependent on either the integration or the derivation of the velocity profile, 
the following velocity profile is employed:

$$
\frac{u_{x}}{u_{e}}=1-\left(1+A(H) \frac{y}{\delta}\right)\left(1-\frac{y}{\delta}\right)^{p(H)-1}
$$

$\delta$ is the dynamic boundary layer thickness. The functions $A$ and $p$ are presented in [1]. Secondly the closure relation proposed by Cousteix [5] is used, $g(H)$ being defined in the aforementioned publication.

$$
\frac{C_{f}}{2} \operatorname{Re}_{\theta_{x}}=g(H) \text { with } \operatorname{Re}_{\theta_{x}}=\frac{u_{e} \theta_{x}}{v}
$$

Further details on the dynamic method can be found in the article of C.Bayeux [1]. The main inputs for the thermalboundary-layer resolution methods exposed hereafter are the friction and dissipation coefficient for the first method (section IV] and the velocity profile for the second method (section $\mathrm{V}$. Notice that other approaches than the method of C. Bayeux could be used to provide these inputs.

\section{Thermal boundary solution using a single equation integral method}

A first method is presented here for the resolution of the thermal boundary layer in an integral way similar to the one exposed in section [IIIfor the resolution of the dynamic boundary layer. This method was already presented in [4] and used successfully for the prediction of the heat transfer coefficient in the laminar area of airfoils without thermal ice protection systems (isothermal wall boundary condition could be used).

\section{A. Derivation of the integral equation}

The flow dynamics is assumed to be known and all the relevant quantities (namely the external velocity $u_{e}$ and the external temperature $T_{e}$ ) at the edge of the boundary layer are known. Over low temperature variations (typically less than $100 \mathrm{~K}$ ), the heat capacity can be considered constant and the enthalpy can be written $h=c_{p} T$ allowing to use the static temperature as the primary variable. On the one hand the two dimensional unsteady energy equation can be written inside the boundary layer (under the assumption that the density variations can be neglected):

$$
\frac{\partial T}{\partial t}+u_{x} \frac{\partial T}{\partial x}+u_{y} \frac{\partial T}{\partial y}=-\frac{1}{\rho c_{p}} \frac{\partial \Phi}{\partial y}+\frac{1}{\rho c_{p}}\left(u_{x} \frac{\partial p}{\partial x}\right)+\frac{1}{\rho c_{p}}\left(\tau_{x y} \frac{\partial u_{x}}{\partial y}\right)
$$

where $\Phi$ is the normal heat flux, $p$ is the pressure and $\tau_{x y}$ is the viscous shear stress. On the other hand the continuity equation writes:

$$
\frac{\partial u_{x}}{\partial x}+\frac{\partial u_{y}}{\partial y}=0
$$

Using both previous equations (6), (5), the following equation (7) is then integrated in the normal direction to the wall over the whole thickness of the thermal boundary layer.

$$
T_{e} \times[\text { continuity equation (6)] }- \text { [energy equation (5)] }
$$

Assuming the fluid in the steady outer flow to be inviscid with a constant total enthalpy, the integral energy equation reads under the conservative form:

$$
\frac{\partial\left(\left(T_{e}-T_{p}\right) \delta_{1 T}\right)}{\partial t}+\nabla \cdot\left(\left(T_{e}-T_{p}\right)\left|u_{e}\right| \theta_{T}\right)=-\frac{\phi_{w}}{\rho c_{p}}-\frac{1}{c_{p}}\left|u_{e}\right|^{3} C_{D}
$$

using the following quantities:

$$
\begin{aligned}
& \left(T_{e}-T_{p}\right) \delta_{1 T}=\int_{0}^{\infty}\left(T_{e}-T\right) d y \\
& \left(T_{e}-T_{p}\right)\left|u_{e}\right| \theta_{T}=\int_{0}^{\infty} u_{x}\left(T_{e}-T\right) d y \\
& \phi_{w}=-k\left(\frac{\partial T}{\partial y}\right)_{y=0} \\
& C_{D}=\frac{1}{\rho\left|u_{e}\right|^{3}} \int_{0}^{\infty} \tau_{x y} \cdot \frac{\partial u_{x}}{\partial y} d y
\end{aligned}
$$


The term $C_{D}$ describing the viscous dissipation in the boundary layer is already known because it was computed during the calculation of the dynamic boundary layer. This scalar equation has too many unknowns. As a consequence it must be closed by linking all the unknowns to a single one, the solved primary variable $\left(T_{e}-T_{p}\right) \delta_{1 T}$.

\section{B. Presumed temperature profile and closure relation}

As stated above the aim of using a closure relation is to make all the aforementioned quantities (9) depend solely on $\left(T_{e}-T_{p}\right) \delta_{1 T}$. One way to do so is by assuming that the temperature in the boundary layer is well described by a specific profile completely defined thanks to the available quantities. Only laminar flows have been investigated so far since the temperature profile of a turbulent flow is different. The supposed temperature profile writes as follows:

$$
T_{e}-T=\Theta(y)=\left(C+D \frac{y}{\delta_{T}}\right)\left(1-\frac{y}{\delta_{T}}\right)^{q-1}
$$

with $\delta_{T}$ being the thermal boundary layer thickness and where the constants $C$ and $D$ are given by:

$$
\left\{\begin{aligned}
& C=\frac{-\left(8(q-1) v\left(\phi_{0}-h_{0} \Theta_{0}\right)+\rho\left|u_{e}\right|^{4} C_{f}^{2} \delta_{T}\right) \delta_{T}}{4(q-1) v\left(q k+2 h_{0} \delta_{T}\right)} \\
& D\left.=\frac{-\left(4(q-1)(q-2) k v\left(\phi_{0}-h_{0} \Theta_{0}\right)+\rho \mid u_{e}{ }^{4} C_{f}^{2}\right.}{4} h_{0} \delta_{T}^{2}+(q-1) k \rho\left|u_{e}\right|^{4} C_{f}^{2} \delta_{T}\right) \delta_{T} \\
& 4(q-1) k v\left(q k+2 h_{0} \delta_{T}\right)
\end{aligned}\right.
$$

where $v$ is the kinematic viscosity. This temperature profile has been designed by assuming that it could be described by a $q$ degree polynomial in $\frac{y}{\delta_{T}}$. The coefficients $C$ and $D$ have been obtained using the $q+1$ conditions listed below.

$$
\begin{aligned}
& \text { in } y=\delta_{T} \quad \Theta=0 \\
& \text { in } y=0-k \frac{\partial T}{\partial y}=\phi_{0}+h_{0}\left(T_{0}-T\right) \text { thus } k \frac{\partial \Theta}{\partial y}=\phi_{0}+h_{0}\left(\Theta-\Theta_{0}\right) \\
& \text { in } y=0 \quad \frac{\partial^{2} T}{\partial y^{2}}=-\frac{\rho\left|u_{e}\right|^{4} C_{f}^{2}}{4 k v} \text { thus } \frac{\partial^{2} \Theta}{\partial y^{2}}=\frac{\rho\left|u_{e}\right|^{4} C_{f}^{2}}{4 k v}
\end{aligned}
$$

with $\Theta_{0}=T_{e}-T_{0}$. The remaining conditions are obtained by imposing all the derivatives up to the $(q-2)$ order in $y=\delta_{T}$ to be zero:

$$
\text { in } y=\delta_{T} \quad \frac{\partial^{j} T}{\partial y^{j}}=0 \quad \text { thus } \quad \frac{\partial^{j} \Theta}{\partial y^{j}}=0 \quad \forall j \leq q-2, j \in \mathbb{N}
$$

The condition $12 \mathrm{c}$ is obtained by writing the local energy equation in $y=0$

$$
0=-\left(\frac{\partial \phi}{\partial y}\right)_{y=0}+\mu\left(\frac{\partial u_{x}}{\partial y}\right)_{y=0}^{2}
$$

where $\mu$ is the dynamic viscosity. Moreover it is known that $\left(\frac{\partial \phi}{\partial y}\right)_{y=0}=-k\left(\frac{\partial^{2} T}{\partial y^{2}}\right)_{y=0}$ and $\left(\frac{\partial u_{x}}{\partial y}\right)_{y=0}=\frac{\left|u_{e}\right|^{2} C_{f}}{2 v}$

This relation involves the friction coefficient which is known thanks to the dynamic boundary layer calculation.

The degree $q$ of the polynomial is obtained by the analysis of solutions obtained for self-similar flows of the Falkner-Skan type for which the external velocity can be written $u_{e}(x)=C x^{m}$, with $C$ and $m$ being two constants. The velocity is supposed low enough for the dissipation term to be negligible. Furthermore the physical properties of the fluid are supposed independent of the temperature. Under those assumptions, almost exact solutions for the temperature profile can be obtained [6]. In addition, for fixed values of the Prandtl number $P r$ and the shape factor $H$ (which is directly linked to the external pressure gradient in these types of flow), the following relation is true:

$$
\operatorname{StPrRe} e_{x}^{1 / 2}=\text { constant }
$$

The Stanton number is defined as: $S t=\frac{\phi_{w}}{\rho_{e}\left|u_{e}\right| c_{p}\left(T_{w}-T_{e}\right)}$. For two values of the shape factor and $\operatorname{Pr}=0.7$, calculations have been carried out with different values of $q$ until the almost exact solution has been computed. The values of $H$ used are the ones referring to a flat plate and a stagnation point in Kays and Crawford's book [6]. A linear approximation is used to link $q$ to $H$. Besides, $q$ has to remain greater than 2 to get a correct behaviour at the edge of the boundary layer. Thus an arbitrary threshold of 2.01 has been used. In the end, it yields the following relation between $q$ and $H$ :

$$
q(H)=\max [(-1.181319 H+6.313094),(2.01)]
$$


Using the now completely defined temperature profile (10), (11) and the relation $9 \mathrm{a}$, as well, it can be inferred that:

$$
\delta_{1 T}=\frac{C(q+1)+D}{q(q+1)} \delta_{T}
$$

This links the thermal boundary layer thickness to the primary variable. The system $(8)$ is thus completely determined. Indeed, the term $\left(T_{e}-T_{p}\right)\left|u_{e}\right| \theta_{T}$ is now ready to be obtained by a simple numerical integration of the temperature times the velocity. However, it must be mentioned that equation (17) has to be solved numerically to infer $\delta_{T}$ from $\delta_{1 T}$, because $C$ and $D$ both depend on $\delta_{T}$, which sometimes may cause some issues.

\section{Thermal boundary layer solution using a modal integral method}

A second more accurate method is proposed in this section for the resolution of the thermal boundary layer. Indeed, the integral method described in section[IV] exhibits some limitations, which are expected to be fixed by the new method based on a Galerkin approach and therefore with more degrees of freedom. The first limitation is the aforementioned difficulty for solving equation (17) in some cases. The second limitation will clearly appear in section VI dedicated to results and discussion. The method of section IV is intrinsically ill-suited for non-uniform thermal wall conditions. It is believed that this problem stems from the fact that the method is not able to take sufficient account of history effects, in particular because the conditions used to close the temperature profile are only local. The proposed new method is of the Galerkin type and allows instead to close the temperature profile by solving additional transport equations. It is therefore expected that history effects will be much better taken into account. In addition, the number of degrees of freedom, and thus the expected accuracy, can be easily increased. In addition, it remains of the integral type, which avoids the need for a mesh in the direction normal to the wall.

\section{A. General principles of the modal integral method}

This integral method being of the Galerkin type, it consists in solving a weak formulation of the problem by projecting the solution on a basis of functions. The solution here is the temperature difference $T_{e}-T=\theta$ as it allows to express more naturally the boundary layer equations than using directly the temperature. First of all, the temperature $T_{e}-T=\theta$ is searched in the space $E_{N}$ of functions $\varphi$ of the following form :

$$
\begin{aligned}
\theta(t, x, y) & =\sum_{j=1}^{N} \Theta_{j}(t, x) \varphi_{j}\left(y, \delta_{T}(x, t)\right) \\
\forall y \leq \delta_{T}, \varphi_{j}\left(y, \delta_{T}(x, t)\right) & =P_{j}(\hat{y}) W(\hat{y}) \\
\forall y>\delta_{T}, \varphi_{j}\left(y, \delta_{T}(x, t)\right) & =0 \\
\hat{y} & =y / \delta_{T}
\end{aligned}
$$

Where the $\Theta_{j}$ are the degrees of freedom of the method and correspond to the components of $\theta$ in the basis $\varphi_{1}, \ldots, \varphi_{N}$. The functions $\varphi_{1}, \ldots, \varphi_{N}$ are $N$ linearly independent shape functions defining a basis of $E_{N}$. The $P_{j}$ are degree $j$ polynomials in $\hat{y}$ that will be defined hereafter. The weighting function $W$ allows the boundary conditions at the edge of the boundary layer to be taken into account. $W$ is defined as $W(\hat{y})=(1-\hat{y})^{q}$. This specific choice of function $W$ stems from the previous experience gained from the aforementioned method (section [IV]). This function $W$ multiplied by a degree one polynomial described fairly accurately the profile of $T_{e}-T$ in the boundary layer for an uniformly imposed wall temperature. The exponent $q$ is not defined the same way as for the previous method and will be determined further.

Retaining the assumption of the previous method that air specific heat capacity variations with temperature are supposed to be negligible over the range of temperatures considered for icing applications, the incompressible enthalpy equation 5 in the boundary layer can be expressed with the temperature. As a reminder, it reads:

$$
\frac{\partial T}{\partial t}+u_{x} \frac{\partial T}{\partial x}+u_{y} \frac{\partial T}{\partial y}=-\frac{1}{\rho c_{p}} \frac{\partial \phi}{\partial y}+\frac{1}{\rho c_{p}}\left(u_{x} \frac{\partial p}{\partial x}\right)+\frac{1}{\rho c_{p}}\left(\tau_{x y} \frac{\partial u_{x}}{\partial y}\right)
$$

Out of the boundary layer by definition: $T_{i e}=c t e$ as a consequence: 


$$
u_{x} \frac{d T_{i e}}{d x}=0
$$

hence considering an incompressible flow:

$$
u_{x} \frac{d T_{e}}{d x}=-\frac{1}{\rho c_{p}} u_{x} \frac{d}{d x}\left(\frac{u_{e}^{2}}{2}\right)
$$

Under the incompressible and irrotational assumption, $p_{e}+1 / 2 \rho V_{e}^{2}=c t e$ and knowing that under the boundary layer assumption $p(x, y)=p_{e}(x)$ :

$$
u_{x} \frac{d T_{e}}{d x}=-\frac{1}{\rho c_{p}} u_{x} \frac{d p_{e}}{d x}=-\frac{1}{\rho c_{p}} u_{x} \frac{d p}{d x}
$$

By definition, $T_{e}$ is independent of $y$ thus:

$$
\vec{V} \cdot \overrightarrow{g r a d} T_{e}=-\frac{1}{\rho c_{p}} \vec{V} \cdot \overrightarrow{\operatorname{grad} p}
$$

Equation 23 along equation 19 yields:

$$
\frac{\partial T}{\partial t}+\vec{V} \cdot \vec{\nabla} T=-\frac{1}{\rho c_{p}} \frac{\partial \phi}{\partial y}+\vec{V} \cdot \vec{\nabla} T_{e}+\frac{1}{\rho c_{p}}\left(\tau_{x y} \frac{\partial u_{x}}{\partial y}\right)
$$

Since the outer flow is steady and by definition $\frac{\partial T_{e}}{\partial t}=0$ it becomes:

$$
\frac{\partial \theta}{\partial t}+\vec{V} \cdot \vec{\nabla} \theta=\frac{1}{\rho c_{p}} \frac{\partial \phi}{\partial y}-\frac{1}{\rho c_{p}}\left(\tau_{x y} \frac{\partial u_{x}}{\partial y}\right)
$$

The integral system of equations is obtained by multiplying equation 25 by a second basis of projection functions $\psi_{i}$, which will also be defined further, and then integrating to the infinity.

$$
\int_{0}^{\infty} \psi_{i}(y)\left(\frac{\partial \theta}{\partial t}+\vec{V} \cdot \vec{\nabla} \theta\right) d y=\int_{0}^{\infty} \psi_{i}(y)\left(\frac{1}{\rho c_{p}} \frac{\partial \phi}{\partial y}-\frac{1}{\rho c_{p}}\left(\tau_{x y} \frac{\partial u_{x}}{\partial y}\right)\right) d y
$$

The temperature $\theta$ is then replaced by the assumed shape of equation (18). As a consequence, by definition $\theta=0$ for $y>\delta_{T}$ and the flow is supposed to show no dissipation outside of the dynamic boundary layer thus $\tau=0$ for $y>\delta$. The system may then be written in a compact form:

$$
\begin{aligned}
& \partial_{t} \mathbf{M}+\partial_{x} \mathbf{F}=\mathbf{S} \\
& \mathbf{S}=\mathbf{\Phi}+\mathbf{Q}^{y}+\hat{\mathbf{D}}+\dot{\Psi}^{x}+\dot{\boldsymbol{\Psi}}^{t} \\
& \mathbf{F}=\sum_{j=1}^{N} \Theta_{j} \int_{0}^{\delta_{T}} u_{x}(y) \boldsymbol{\psi}(y) \varphi_{j}(y) d y
\end{aligned}
$$

The quantity $\mathbf{Q}$ describes a flux term while $\mathbf{S}$ are the source terms and $\mathbf{M}$ is the unsteady term. As a consequence, this equation is an unsteady equation of conservation and can be solved using the Finite-Volume method. Notice that since some steady-flow assumptions were used in the derivation of the method, the system (27a) does not represent the unsteady problem. But it can be used to solve the steady problem by converging the unsteady sytem (27a) to steady-state. The link between the temporal derivative term and the solved quantity $\Theta$ (the vector of $\Theta_{j}$ ) is written:

$$
\begin{aligned}
& A=\left[a_{i, j}\right]=\left[\int_{0}^{\delta_{T}} \psi_{i}(y) \varphi_{j}(y) d y\right] \\
& \mathbf{M}=A \boldsymbol{\Theta}
\end{aligned}
$$

The source term describing the heat conduction through the thermal boundary layer reads: 


$$
\Phi_{i}=\int_{0}^{\delta_{T}} \frac{1}{\rho c_{p}} \frac{\partial \phi}{\partial y} \psi_{i} d y
$$

Using the Fourier law, the heat flux reads: $\phi=k \frac{\partial \theta}{\partial y}$, with $k$ being the thermal conductivity, and by replacing $\theta$ by its definition (18). :

$$
\Phi_{i}=\alpha \sum_{j=1}^{N} \Theta_{j} \int_{0}^{\delta_{T}} \frac{\partial^{2} \varphi_{j}}{\partial y^{2}} \psi_{i} d y
$$

where $\alpha=\frac{k}{\rho c_{p}}$. Then, by performing an integration by parts and knowing that for $y=\delta_{T}, \forall j, \varphi_{j}=0$ :

$$
\Phi_{i}=-\alpha \sum_{j=1}^{N} \Theta_{j}\left(\int_{0}^{\delta_{T}} \frac{\partial \varphi_{j}}{\partial y} \frac{\partial \psi_{i}}{\partial y} d y+\left.\frac{\partial \varphi_{j}}{\partial y}\right|_{y=0} \psi_{i}(0)\right)
$$

The source term describing the viscous dissipation reads:

$$
\begin{aligned}
& \hat{D}_{i}=-\int_{0}^{\delta} \dot{D}(y) \psi_{i}(y) d y \\
& \dot{D}(y)=\frac{1}{\rho c_{p}}\left(\tau_{x y} \frac{\partial u_{x}}{\partial y}\right)
\end{aligned}
$$

The source term describing the heat flux convected by the normal velocity reads:

$$
\dot{Q}_{i}^{y}=-\int_{0}^{\delta_{T}} \frac{\partial}{\partial y}\left(u_{y} \theta\right) \psi_{i} d y
$$

Performing once more an integration by parts and replacing $\theta$ by its definition (18), knowing that $\theta_{e}=0$ and $u_{y}(0)=0$.

$$
\dot{Q}_{i}^{y}=\sum_{j=1}^{N} \Theta_{j} \int_{0}^{\delta_{T}} \varphi_{j} u_{y} \frac{\partial \psi_{i}}{\partial y} d y
$$

While this term describes a convective flux, the fact that the energy equation has been integrated over the vertical direction turns it into a source term rather than a flux term from a numerical point of view. The mathematical meaning of this term is a redistribution term by the vertical speed over the space of functions used $E_{N}$. In other words, the fact that a non-zero vertical speed exists bends the temperature profile which, in a modal approach such as this, translates into a change of the weight of each mode due to an additional source term.

Nevertheless, the normal velocity is not known since not calculated while solving the dynamic boundary layer. As a matter of fact it can be easily obtained through the continuity equation and knowing the longitudinal velocity profile in the boundary layer.

$$
u_{y}(Y)=-\int_{0}^{Y} \frac{\partial u_{x}}{\partial x} d y
$$

Since the velocity is a constant input data regarding the calculation of the thermal boundary layer, a single calculation of the vertical velocity field is needed.

Finally the two terms $\dot{\Psi}_{i}^{x}$ and $\dot{\Psi}_{i}^{t}$ appear in case the function $\psi$ has any dependence on space or time:

$$
\begin{aligned}
\dot{\Psi}_{i}^{x} & =\sum_{j=1}^{N} \Theta_{j} \int_{0}^{\delta_{T}} u_{x}(y) \varphi_{j}(y) \frac{\partial \psi_{i}}{\partial x} d y \\
\dot{\Psi}_{i}^{t} & =\sum_{j=1}^{N} \Theta_{j} \int_{0}^{\delta_{T}} \varphi_{j}(y) \frac{\partial \psi_{i}}{\partial t} d y
\end{aligned}
$$




\section{B. Choice of the bases and expression of the boundary conditions}

The choice of the functions $\varphi$ and $\psi$ is a key part of the process and must be done with care since results may greatly depend on it. Regarding the functions of projection $\psi$, polynomial functions in either $y$ or $\hat{y}$ have been investigated. The latter is both mathematically and numerically troublesome (because of the dependency of $\hat{y}=y / \delta_{T}$ with respect to $\mathrm{x}$ and $\mathrm{t}$ through $\delta_{T}$ ) and yields inaccurate results as well. As such, this option has been discarded. The following basis is used.

$$
\begin{aligned}
\psi_{i} & =\frac{y^{i-1}}{C_{q, i}} \\
C_{q, i} & =\int_{0}^{1}(1-y)^{q} y^{i-1} d y
\end{aligned}
$$

The use of the $C_{q, i}$ coefficient allows a better conditioning of the system which would otherwise result in quickly growing coefficients with the number of modes. Using this definition of $\psi$, it has no dependence over time and space as long as the exponent $q$ does not, which entirely nullifies the two aforementioned sources terms (eq. 36)

The $q$ exponent used both in the weight function $W$ and in the $\psi$ functions is chosen to be equal to $N$, the number of modes. It has been established that the results are not strongly dependent on $q$ as long as it is high enough. Indeed, the floor of $q$ indicates the number of zero derivatives of the temperature profile on the upper edge of the boundary layer. Should this number be lower than the degree of the polynomial used, the temperature profile can experience non zero oscillations on the border of the boundary layer, which is not in agreement with the boundary layer assumption. On the other hand, too high $q$ exponent results in according not enough weight to the upper part of the boundary layer leading to a slight loss of informations and thus yielding less accurate results.

The basis $\varphi$ is then deduced by imposing that the matrix $A$ is triangular inferior (eq. 28b) and that the diagonal terms $A_{i, i}$ are equal to $\left(\delta_{T}\right)^{i}$. This specific choice of $A$ ensures that it is always invertible, meaning that $\Theta$ can always be deduced from the unsteady term $\mathbf{M}$ and $A^{-1}$. Those conditions on $A$ imply that $\varphi_{j}$ has $j$ non-zero coefficient. The degree of the polynomials are yet to be defined. This is done by considering the boundary layer conditions. For the sake of simplicity, the degrees are chosen so that, for an imposed wall temperature, only the first component is used in the relation between the thermal boundary layer thickness and the unsteady term (this will be addressed a little further). This means that the $P_{1}$ polynomial is a constant while the remaining $P_{j}$ are polynomials of degree $j$ with only the constant coefficient being zero. All this leads to the following expression of $\varphi$.

$$
\begin{aligned}
j=1, \varphi_{1}\left(y, \delta_{T}\right) & =B_{1,1} * C_{q, 1} W(\hat{y}) \\
\forall j>1, \varphi_{j}\left(y, \delta_{T}\right) & =\sum_{k=1}^{j} B_{j, k} \hat{y}^{k} * C_{q, j} W(\hat{y})
\end{aligned}
$$

The matrices $B$ depend on $q$ but are time independent. Their role is to ensure that the matrix $A$ is of the desired form. Since $q$ has been defined as space independent, so are the $B$ which means there is a single $B$ matrix. Otherwise it would just mean that the matrices $B$ are to be calculated for each point of the profile. The matrix $B$ is defined through a sum of recursive functions which appear too complex to solve analytically but easily solved numerically. The conditions chosen for $A$ state for $j=1$ :

$$
A_{1,1}=\int_{0}^{\delta_{T}} B_{1,1}(1-\hat{y})^{q} d y=\delta_{T}
$$

Therefore $B_{1,1}=q+1$

On the other hand, $\forall j>1$ :

$$
\begin{aligned}
& \forall j>i, A_{i, j}=\int_{0}^{\delta_{T}} \sum_{k=1}^{j} B_{j, k} \frac{C_{q, j}}{C_{q, i}} \hat{y}^{k} y^{i-1}(1-\hat{y})^{q} d y=0 \\
& \forall j=i, A_{i, i}=\int_{0}^{\delta_{T}} \sum_{k=1}^{i} B_{i, k} \hat{y}^{k} y^{i-1}(1-\hat{y})^{q} d y=\left(\delta_{T}\right)^{i}
\end{aligned}
$$


It can be shown through recursive reasoning that:

$$
\int_{0}^{\delta_{T}} y^{i}\left(1-y / \delta_{T}\right)^{q} d y=\frac{i !\left(\delta_{T}\right)^{i+1}}{\prod_{k=1}^{i+1}(q+k)}
$$

Knowing that, the matrix $A$ can be reshaped

$$
\begin{aligned}
A_{i, j} & =\sum_{k=1}^{j} B_{j, k} \frac{C_{q, j}}{C_{q, i}} F_{k, i}\left(\delta_{T}\right)^{i} \\
F_{k, i} & =\frac{(i+k-1) !}{\prod_{l=1}^{i+k}(q+l)}
\end{aligned}
$$

Injecting the condition (42) into the left hand of [40, $\forall j \geq i$, the $B$ matrix is defined such as $\forall j \in[2, N]$ (using the Kronecker symbol):

$$
\sum_{k=1}^{j} B_{j, k} F_{k, i} \frac{C_{q, i}}{C_{q, j}}=\delta_{i, j}
$$

Subsequently, $\forall i=j$

$$
\sum_{k=1}^{j} B_{j, k} F_{k, j}=1
$$

which simply becomes $\forall k \leq j, B_{j, k}=F_{k, j}^{-1}$. Thus, the system is completely defined. Only $\delta_{T}$ is time dependent, meaning considering the calculation of $A^{-1}$, the reverse of $\sum_{k=1}^{j} B_{j, k} \frac{C q_{j}}{C q_{i}} F_{k, i}$ is computed only once while $\left(\delta_{i, j} \delta_{T}^{i}\right)^{-1}$ is computed at each iterations. The yet unknown $\delta_{T}$ is computed using the thermal boundary conditions. For an imposed wall temperature using the temperature profile 18 and the definition of the functions $\varphi$ (eq $38 \mathrm{~b}$ it follows, since only the first function $\varphi_{1}$ has a component independent of $y$.

$$
\text { in } y=0, \quad \theta=\sum_{j=1}^{N} \Theta_{j} \varphi_{j}(0)=\varphi_{1}(0) \Theta_{1}=C_{q, 1} B_{1,1} \Theta_{1}=\theta_{w}
$$

Moreover considering $28 \mathrm{~b}$ and by construction of the lower triangular matrix $A:\left[A^{-1} \mathbf{M}\right]_{1}=\frac{M_{1}}{\delta_{T}}$ :

$$
\Theta_{1}=\frac{M_{1}}{\delta_{T}}
$$

As a result, since $B_{1,1}=q+1$ and $C_{q, 1}=(q+1)^{-1}$ :

$$
\delta_{T}=\frac{M_{1}}{\theta_{w}}
$$

The equation 47 ensures the unicity of the solution yielded by the method for an imposed wall temperature.

\section{Finite volume formulation and numerical integration}

The system of equations $27 \mathrm{a}$ can be written in the following manner:

$$
\frac{\partial(\mathbf{M})}{\partial t}+\tilde{\nabla} \cdot \mathbf{F}(\mathbf{M})=\mathbf{S}(\mathbf{M})
$$

Using the matrix $A$ defined in $28 b$ the components of the flux vector $\mathbf{F}$ can be written:

$$
F_{i}=\sum_{j=1}^{N} A_{j k}^{-1} M_{k} \int_{0}^{\delta_{T}} u_{x}(y) \psi_{i}(y) \varphi_{j}(y) d y
$$


Following a Finite-Volume formulation, the system is integrated over a cell $i$ stretching from $x_{i-1 / 2}$ to $x_{i+1 / 2}$, between time $t^{n}$ and $t^{n+1}$ :

$$
\int_{x_{i-1 / 2}}^{x_{i+1 / 2}} \mathbf{M}\left(x, t^{n+1}\right) d x-\int_{x_{i-1 / 2}}^{x_{i+1 / 2}} \mathbf{M}\left(x, t^{n}\right) d x=-\int_{t^{n}}^{t^{n+1}}\left(\mathbf{F}\left(x_{i+1 / 2}, t\right)-\mathbf{F}\left(x_{i-1 / 2}, t\right)\right) d t+\int_{t^{n}}^{t^{n+1}} \int_{x_{i-1 / 2}}^{x_{i+1 / 2}} \mathbf{S}(x, t) d x d t
$$

Equation 50 can be rewritten in the discrete form as such:

$$
\mathbf{M}_{i}^{n+1}=\mathbf{M}_{i}^{n}-\frac{\Delta t^{n}}{\Delta x_{i}}\left(\mathbf{F}_{i+1 / 2}-\mathbf{F}_{i-1 / 2}\right)+\Delta t^{n} \mathbf{S}_{i}^{n}
$$

where $\mathbf{M}_{i}^{n}$ corresponds to the mean values of the unknowns in the cell $i$ at the time $t^{n}, \mathbf{F}_{i \pm 1 / 2}^{n}$ are the numerical flux at the vertices $i \pm 1 / 2$ at the time $t^{n}$ and $\mathbf{S}_{i}^{n}$ is the discrete source term in cell $i$ :

$$
\begin{gathered}
\mathbf{M}_{i}^{n} \stackrel{\text { def }}{=} \frac{1}{\Delta x_{i}} \int_{x_{i-1 / 2}}^{x_{i+1 / 2}} \mathbf{M}\left(x, t^{n}\right) d x \\
\mathbf{F}_{i+1 / 2}^{n} \stackrel{\text { def }}{=} \frac{1}{\Delta t^{n}} \int_{t^{n}}^{t^{n+1}}\left(\mathbf{F}\left(x_{i+1 / 2}, t\right)\right) d t \\
\mathbf{S}_{i}^{n} \stackrel{\text { def }}{=} \frac{1}{\Delta t^{n} \Delta x_{i}} \int_{t^{n}}^{t^{n+1}} \int_{x_{i-1 / 2}}^{x_{i+1 / 2}} \mathbf{S}(x, t) d x d t
\end{gathered}
$$

As far as the numerical flux is concerned, a first order upwind scheme is used for the expression of the fluxes in order to ensure stability. An Euler explicit method has been employed for the discretization of both the transport term and source terms. This implies a limiting numerical stability condition on the time step. Using an implicit discretization for the source terms would allow to increase the maximum time step and as such will be carried out in a near future. Furthermore increasing the number of modes used further reduces the maximum time step allowed to ensure the stability of the method. The maximum time step also depends on the external aerodynamic and the meshing used to obtain it, more refined mesh tending to reduce the maximum time step. No theoretical condition have been determined for the maximum time step. The local time step is determined through $C F L=\max \left(u_{e}\left(x_{i}\right) d t / d x_{i}\right)<C F L_{\max }(N)$. Typical values for $C F L_{\max }(N)$ are: $C F L_{\max }(2)=0.1, C F L_{\max }(3)=0.05, C F L_{\max }(4)=0.05$ and $C F L_{\max }(5)=0.02$.

All the numerical integrations are performed through a Gauss-Legendre Quadrature which provides the exact solution for polynomials up to degree $2 n-1, n$ being the number of points of integration. The temperature profile and the functions of projection $\psi_{i}$ are polynomials while the velocity profile can be closely approximated by low order polynomials. Thus this integration strategy seems well founded. Using $2 N$ points of integration, $N$ being the number of modes solved, it yields more than a four digit accuracy. In the case of the heat conduction source terms, it only requires integrations of functions with a known primitive therefore the integration is performed analytically. The details of said integration are not displayed here for the sake of conciseness.

The method is carried out as follows. Firstly, $\delta_{T}$ is initialized with the dynamic boundary layer thickness $\delta$. The Reynolds analogy states that both boundary layers thickness are proportional and even equal, with a Prandtl number equal to 1 . While this is is obviously not true (hence the present method) this allows to initialize the boundary layer close to the solution. Then, the first component of the vector $\mathbf{M}$ is initialized thanks to equation $47 \mathrm{knowing} \theta_{w}$, while the rest of the components are zero. Constant coefficients, matrices and the vertical speed are then computed. Afterwards, the time loop is performed, the first step being to deduce $\boldsymbol{\Theta}$ from $\mathbf{M}$ and $\delta_{T}$. The second step is to compute $\mathbf{M}$ for the next time step. Finally $\delta_{T}$ can be computed for the next iteration. Calculation are performed until the $L 2$ norm of the residual drops below a predefined threshold. Finally the wall heat heat flux is computed using the following expression:

$$
\phi_{w}=\left.k \sum_{j=1}^{N} \Theta_{j}(t, x) \frac{\partial \varphi_{j}}{\partial y}\right|_{y=0}
$$




\section{Results}

The two methods previously described are compared to reference solutions calculated by the ONERA code CLICET [7] which uses a 2D mesh and Finite Volume method to solve the Prandtl equations. The comparisons are first carried out on wedge flows with the external velocity being described by: $u_{e}(x)=C x^{m}$. Those cases are investigated because they are representative of boundary layer flows with a local external pressure gradient. The results for isothermal conditions are investigated at first before cases of a wall temperature varying linearly are investigated. In a second time, wing profiles are investigated both with zero and non-zero angles of attack. NACA 0012 and GLC305 profiles are presented under both uniform and non uniform wall temperature. The order of magnitude of the temperatures are chosen so as to be representative of de-icing systems. In the following graphs, "S-BLIM" refers to the single equation integral method introduced in section IV while "M-BLIM" refers to the modal integral method introduced in section $\mathrm{V}$

\section{A. Wedge flow: Isothermal conditions}

Figures 1 through 6 refer to wedge flow cases for which there are the self similar solutions of Falkner-Skan of the dynamic boundary layer. $s=x / L$ is the non dimensional longitudinal coordinate. The external velocity is expressed $U_{e}=c s^{m}$, where $c$ and $m$ are two constants. The pressure gradient is determined by $m$, with negative values of $m$ describing adverse pressure gradients and positive values describing favourable pressure gradients. The situation $m=0$ represents a flat plate with no pressure gradient while the situation $m=1$ represents a stagnation point. The Stanton numbers $(S t)$ are shown along the whole profile and compared to the reference solution. The relative error on the wall heat flux $\left(\left(\phi_{w}-\phi_{w, \text { ref }}\right) / \phi_{w, \text { ref }}\right)$ are also shown along the whole profile. The supposed temperature profiles along the normal to the wall for both methods at a specific location are compared to the reference temperature profile. They are made non dimensional through $\left(T-T_{e}\right) /\left(T_{w}-T_{e}\right)$ while the vertical coordinate is made non dimensional by dividing by the thermal boundary layer thickness of the reference solution, meaning the temperature profile goes from zero to one only for the reference solution. This choice allows to compare the thermal boundary layer thickness while still using non dimensional values. The modal method yields slightly better results for all cases. The modal method with 2 modes is more accurate than the single equation method on all cases but the accelerated flow with $m=0.3333$. Using more modes clearly improves the results for all the accelerated flows $(m=0.3333$ and $m=1.0)$. The temperature profiles show that the modal method with enough modes allows to better capture the upper part of the boundary layer with a thermal boundary thickness closer to the reference solution. The external conditions for the wedge flows are $M_{\infty}=0.25$, $P_{\infty}=80000 P a, T_{\infty}=263.15 K$. 


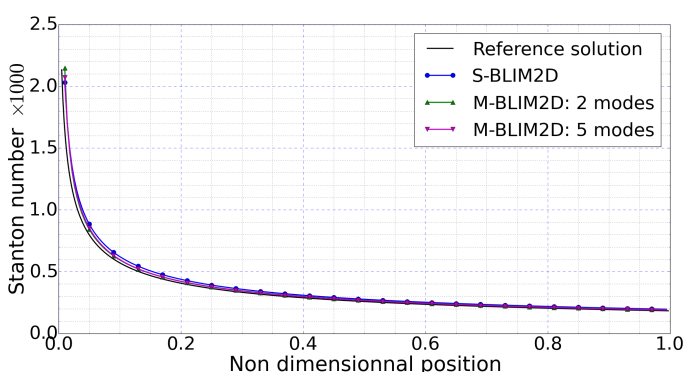

(a) Stanton number

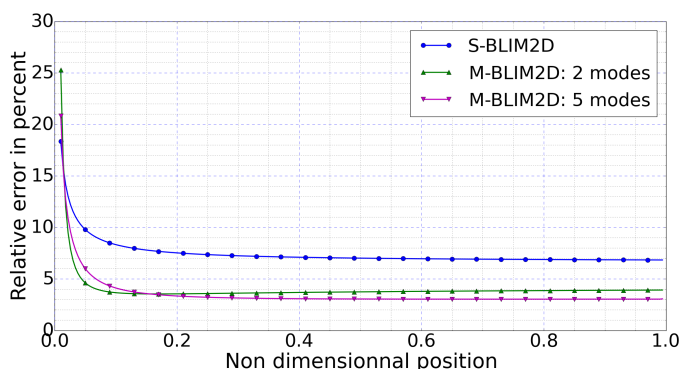

(b) Relative error on the wall heat flux

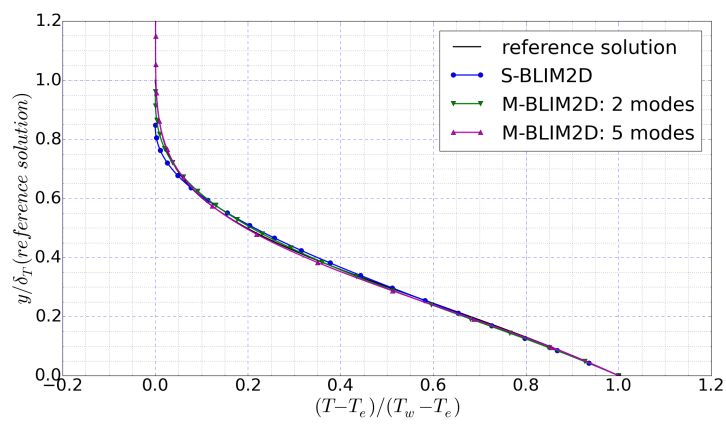

(c) Non dimensional Temperature profile in $s=0.5$

Fig. 1 Flat plate for an imposed wall temperature $T_{w}=273.15 K$. The reference solution thermal boundary layer thickness is used to make the vertical coordinate non dimensional.

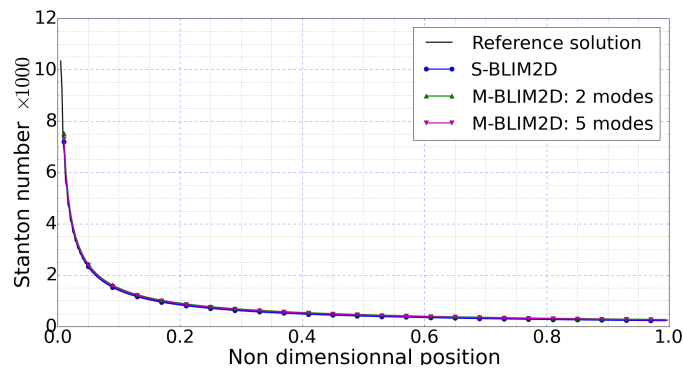

(a) Stanton number

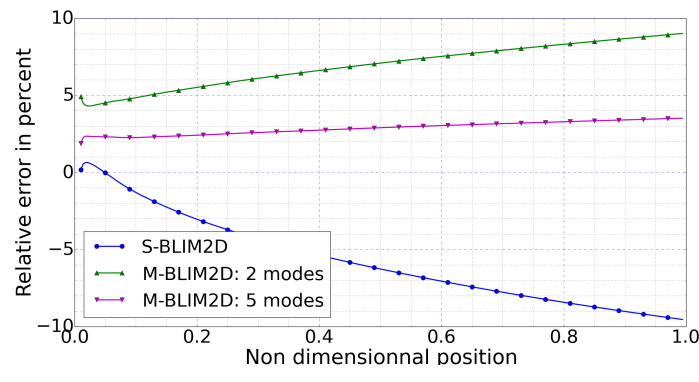

(b) Relative error on the wall heat flux

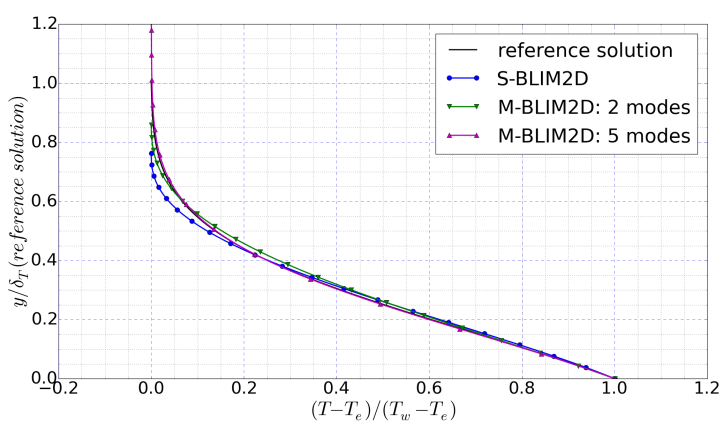

(c) Non dimensional temperature profile in $s=0.5$.

Fig. 2 Accelerated flow, $m=0.3333$, for an imposed wall temperature $T_{w}=273.15 K$. The reference solution thermal boundary layer thickness is used to make the vertical coordinate non dimensional. 


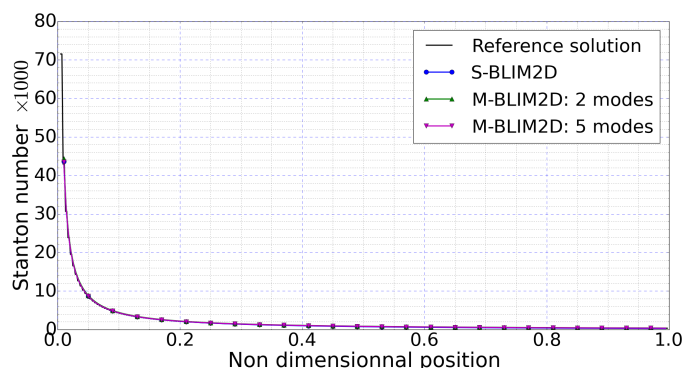

(a) Stanton number

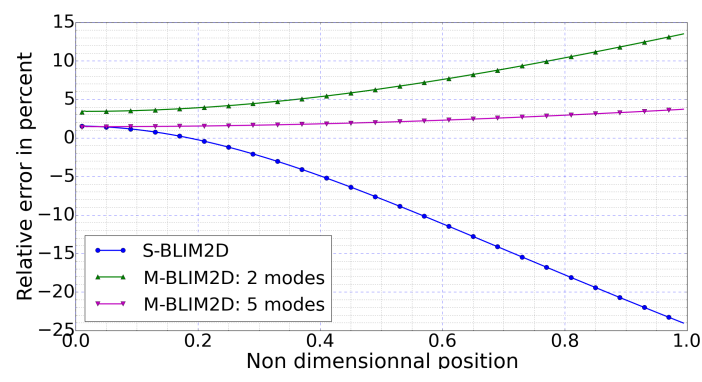

(b) Relative error on the wall heat flux

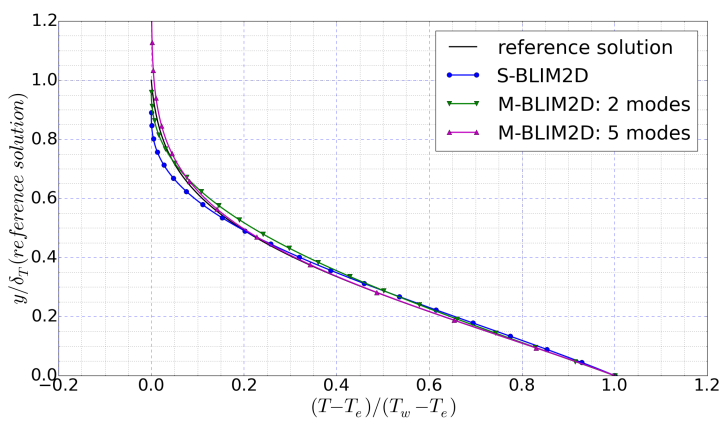

(c) Non dimensional Temperature profile in $s=0.5$

Fig. 3 Stagnation point, $m=1$, for an imposed wall temperature $T_{w}=273.15 K$. The reference solution thermal boundary layer thickness is used to make the vertical coordinate non dimensional.

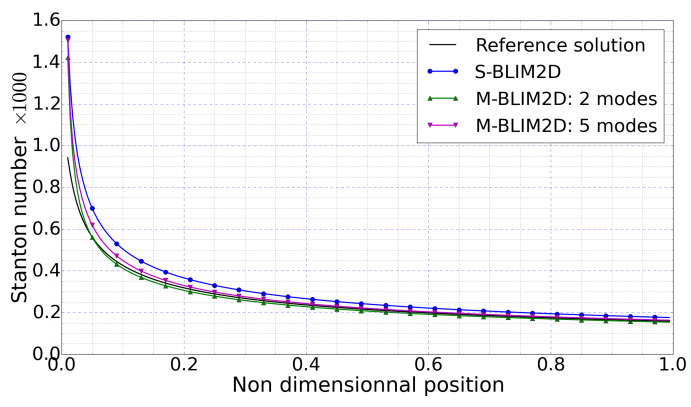

(a) Stanton number

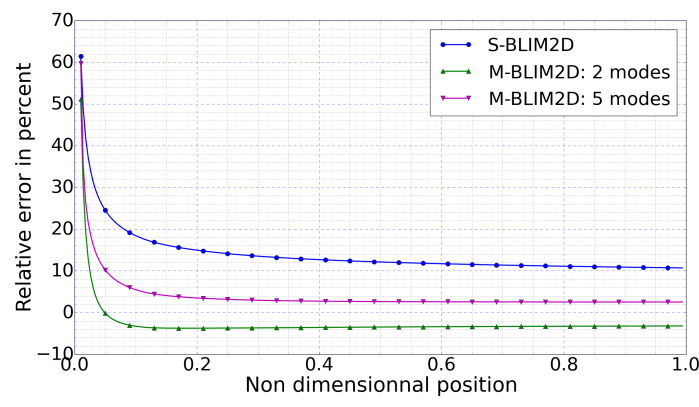

(b) Relative error on the wall heat flux

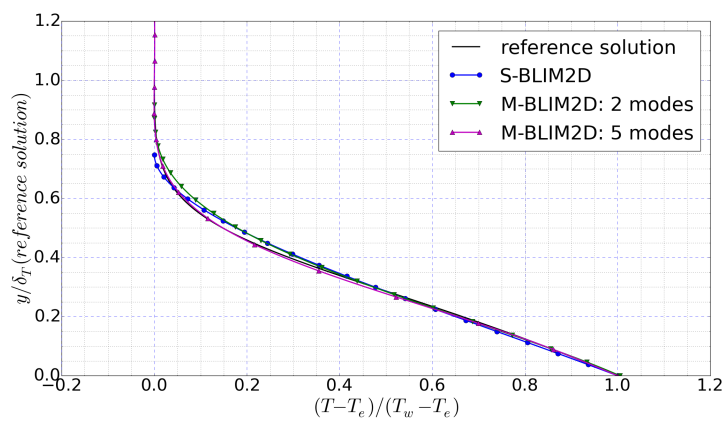

(c) Non dimensional Temperature profile in $s=0.5$

Fig. 4 Decelerating flow, $m=-0.07$, for an imposed wall temperature $T_{w}=273.15 \mathrm{~K}$. The reference solution thermal boundary layer thickness is used to make the vertical coordinate non dimensional. 


\section{B. Wedge flow: Non isothermal conditions}

Figures 5 and 6 refer to accelerated wedge flows with $m=0.3333$ and a wall temperature that varies linearly with the distance to the leading edge. The gradient is $\left|\vec{\nabla} T_{w}\right|=-400 \mathrm{~K} . \mathrm{m}^{-1}$ for figure 5 the wall temperature going from $T_{w}=373.15 \mathrm{~K}$ at $s=0$ to $T_{w}=273.15 \mathrm{~K}$ at $s=1$. The figures $5(\mathrm{a}-\mathrm{b})$ show the $S t$ number. The presence of the wall temperature gradient induces a specific behaviour of the wall heat flux, that is to say the air near the wall is heated through conduction and convected downstream which has a lower wall temperature. At some point, the air near the wall becomes hotter than the wall, thus heating the wall despite the wall being in a cold outer flow. The single equation integral method is incapable of describing this behaviour and quickly departs from the solution. On the contrary the modal method allows to describe accurately the wall heat flux up to $s=0.6$ approximately. Afterwards some discrepancy can be observed depending on the number of modes used. Only two modes fail at capturing the whole area showing a negative wall heat flux. As the number of modes increases, the point at which the wall heat flux starts departing from the solution, is further pushed downstream. The figure 5 (c-d-e) shows the non dimensional temperature profile along the vertical direction for $s=0.4, s=0.6$ and $s=0.8$. The further downstream, the more curvature appears on the temperature profiles and the more the use of the modal method becomes relevant. Figure 5(e) clearly shows that increasing the number of modes used increases the accuracy of the presumed temperature profile. It also shows that the temperature profile of the single equation method is completely off chart in this case.

The case described by figure 6 has a gradient of $\left|\vec{\nabla} T_{w}\right|=+400 K . \mathrm{m}^{-1}$, with the wall temperature going from $T_{w}=273.15 \mathrm{~K}$ at $s=0$ to $T_{w}=373.15 \mathrm{~K}$ at $s=1.0$. The Stanton number described in 6 (a) and the relative error on the flux described in 6 b) show that the modal method yields more accurate results than the one equation method if enough modes are used. The figure 6(c) shows the non dimensional temperature along the vertical direction for $s=0.5 \mathrm{~m}$. The use of several modes allows to capture more accurately the highest part of the temperature profile but does not improve the middle section. Still, considering the significant improvement of the wall heat flux with the modal method, there is no denying that the temperature near the wall is better captured.

The two previous cases show the benefit of the modal method for non uniform imposed wall temperature and the fact that the more modes are calculated, the better the results. 


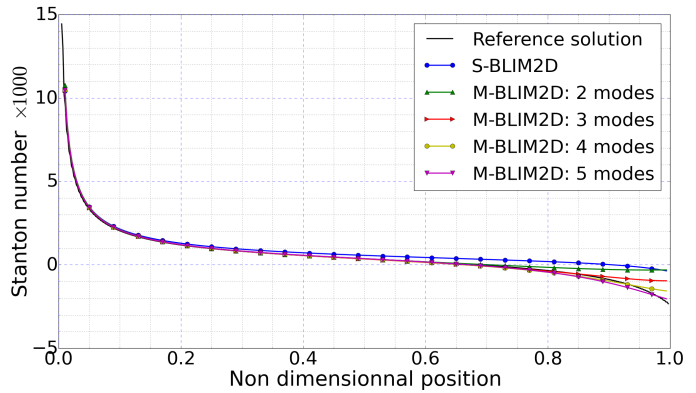

(a) Stanton number

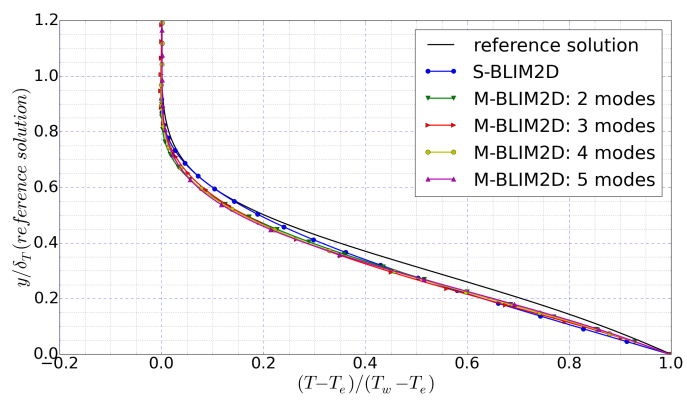

(c) Non dimensional temperature profile in $s=0.4$

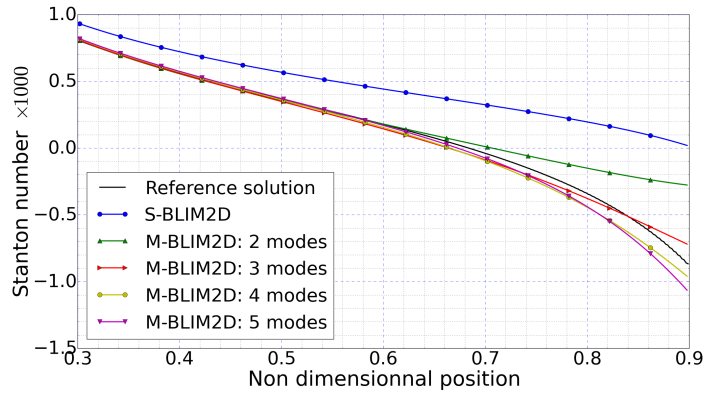

(b) Stanton number, zoom between $s=0.3$ and $s=0.9$

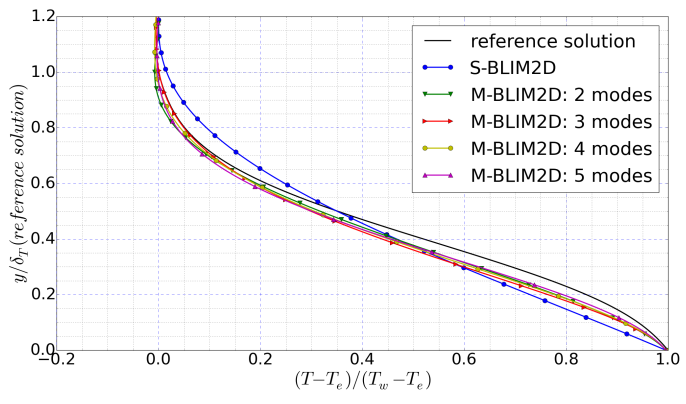

(d) Non dimensional temperature profile in $s=0.6$

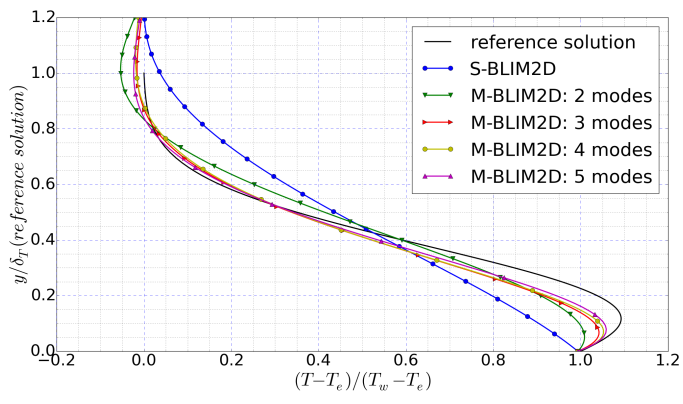

(e) Non dimensional temperature profile in $s=0.8$

Fig. 5 Accelerated flow, $m=0.3333$ for an imposed wall temperature $T_{w}=373.15 \mathrm{~K}$ at $s=0$ and $T_{w}=273.15 \mathrm{~K}$ at $s=1$. The reference solution thermal boundary layer thickness is used to make the vertical coordinate non dimensional. 


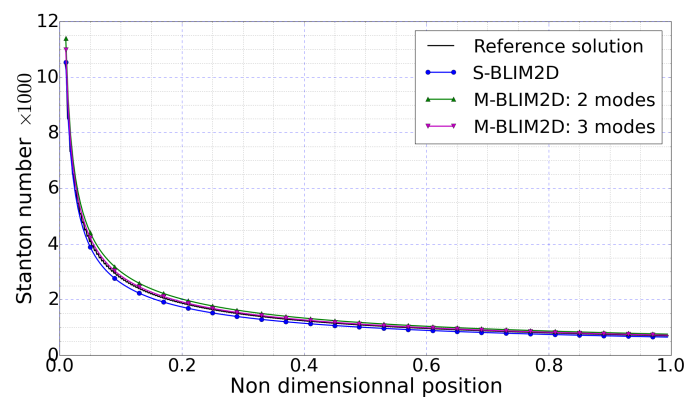

(a) Stanton number

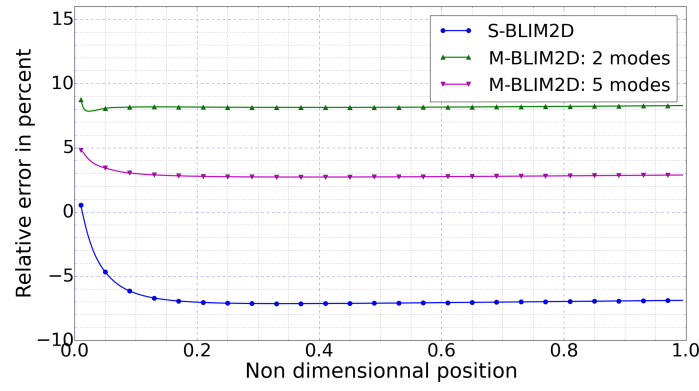

(b) Relative error on the wall heat flux

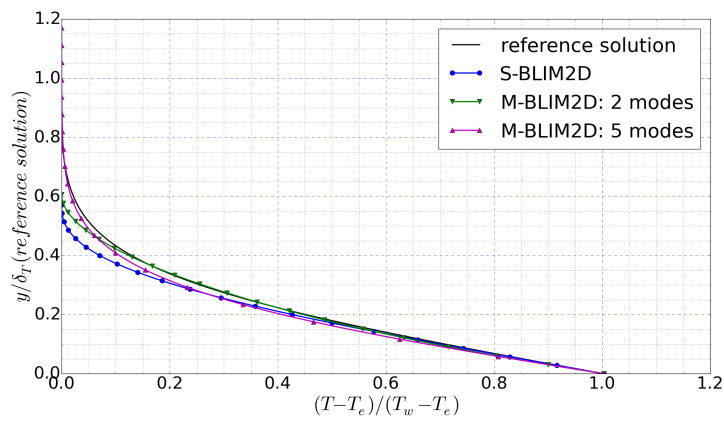

(c) Non dimensional Temperature profile in $s=0.5$

Fig. 6 Accelerated flow, $m=0.3333$ for an imposed wall temperature $T_{w}=373.15 \mathrm{~K}$ at $s=0$ and $T_{w}=273.15 \mathrm{~K}$ at $s=1$. The reference solution thermal boundary layer thickness is used to make the vertical coordinate non dimensional. 


\section{Wing profile: Isothermal conditions}

For all the figures referring to a wing profile, the coordinate $x=0 m$ is the stagnation point. $x>0 m$ refers to the suction side while $x<0 \mathrm{~m}$ is the pressure side. Figures 7 to 9 refer to wing profiles at a constant imposed wall temperature of $T_{w}=303.15 \mathrm{~K}$. Figure 7 refers to a NACA 0012 profile with a $0^{\circ}$ angle of attack and the following external conditions: $M_{\infty}=0.25, P_{\infty}=101325 \mathrm{~Pa}, T_{\infty}=263.15 \mathrm{~K}$. The solution is symmetrical as expected and the solution of both S-BLIM and M-BLIM are within 5\% to the reference solution. 3 modes are sufficient to reach convergence and yield more accurate results than the single equation method. Figure 7 (c-d) show that the modal method does not allow to capture better the upper part of the temperature profile near the stagnation point but does so further downstream.

Figure 8 refers also to a NACA 0012 profile but with a $4^{\circ}$ angle of attack, $x=0 \mathrm{~m}$ still being the position of the stagnation point. The external conditions are: $M_{\infty}=0.3, P_{\infty}=80000 P a, T_{\infty}=263 \mathrm{~K}$. The pressure side shows the same behaviour as the zero incidence case, with both method being under $5 \%$ error with the reference solution. On the other hand, the suction side shows much more discrepancies between the methods. Indeed, the single equation method yields inaccurate results, in particular it fails at reproducing the maximum of wall heat flux, both in terms of position and value. On the other hand, the modal method locates successfully the maximum of the wall heat flux and is more accurate. Using 2 modes yields a large error around the maximum of the wall heat flux while 3 or more modes yields less than a 5\% error. The temperature profiles 8 (c-d) show that while the modal method allows to better capture the top of the boundary layer, it is not the case for the middle section.

Figure 9 refers to the GLC 305 profile under a $4^{\circ}$ incidence and the following external conditions: $M_{\infty}=0.25$, $P_{\infty}=101325 \mathrm{~Pa}, T_{\infty}=263.15 \mathrm{~K}$. The wall heat flux for the pressure side shows the same characteristics as the previous case. On the contrary, while the suction side shows the same key features as the previous case concerning the maximum of wall heat flux, the flow on suction side separates close to the stagnation point. This means that the comparison can only be performed for a short distance. 3 modes are enough to reach convergence and yield more accurate results than the one equation method.

Mesh converging has been carried out on the NACA 0012 profile at $0^{\circ}$ angle of incidence, using evenly spaced meshing. Increasing the number of nodes increases the accuracy of the solution, especially near the stagnation point. However, the method does not converge towards the solution but close to it. Table 1 shows the L2 norm of the error on the wall heat flux versus the number of nodes used for the meshing while figure 10 shows the wall heat flux compared to the reference solution computed by CLICET with highly refined mesh than provides convergence. 3 modes have been used for the calculation depicted by both.

\begin{tabular}{|c|c|}
\hline Number of points & L2 Norm of the error \\
\hline 128 & 0.0827 \\
256 & 0.0279 \\
512 & 0.0120 \\
\hline
\end{tabular}

Table 1 L2 error on the wall heat flux versus the number of nodes used for the meshing 


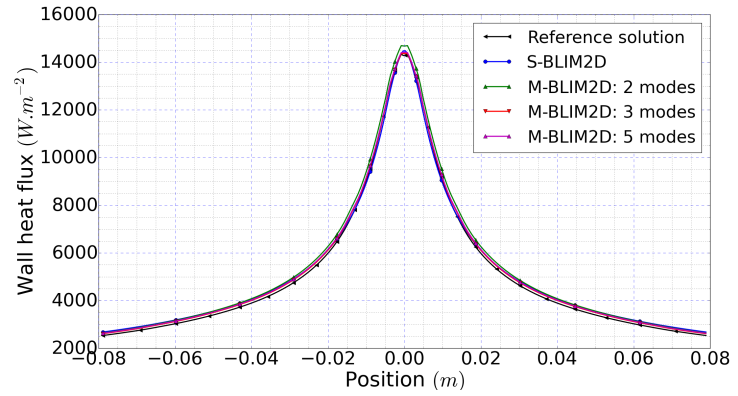

(a) Wall heat flux

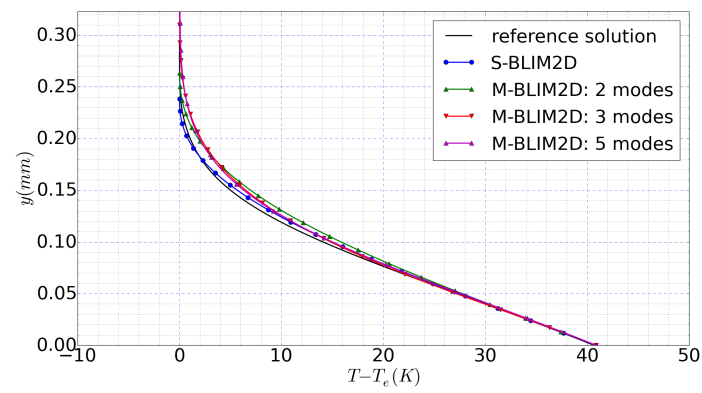

(c) Temperature profile in $x=0.02 m$

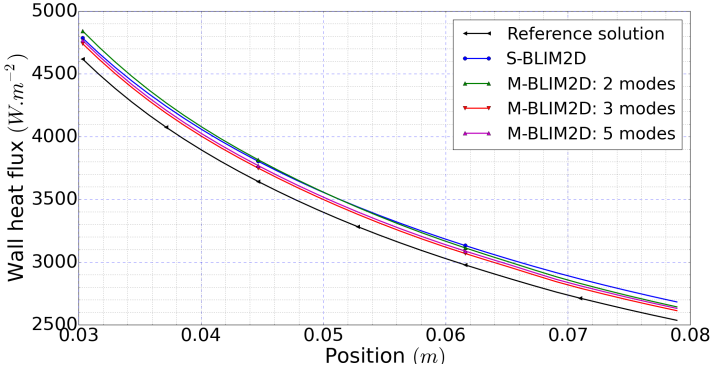

(b) Wall heat flux between $x=0.03 \mathrm{~m}$ and $x=0.08 \mathrm{~m}$ suction side

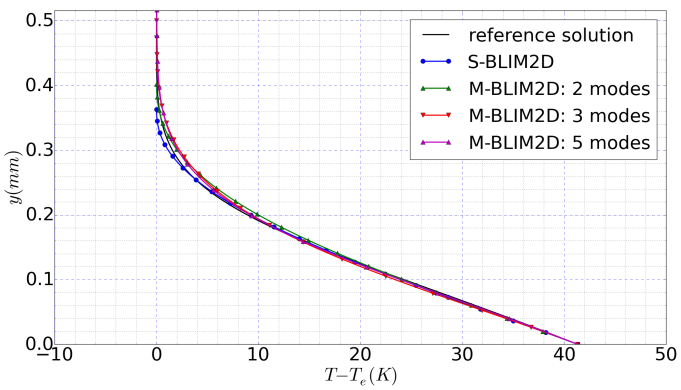

(d) Temperature profile in $x=0.04 m$

Fig. 7 NACA 0012 profile with a $0^{\circ}$ angle of attack and a constant wall temperature of $T_{w}=303.15 K$.

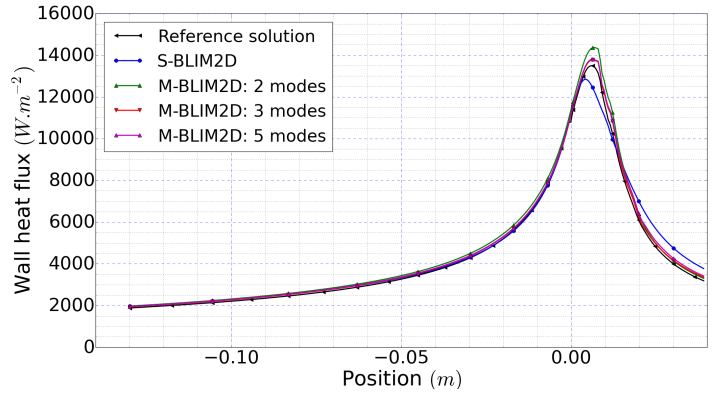

(a) Wall heat flux

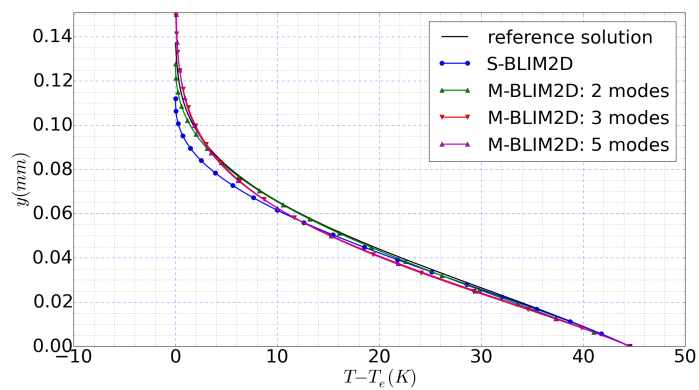

(c) Temperature profile in $x=0.01 \mathrm{~m}$, suction side

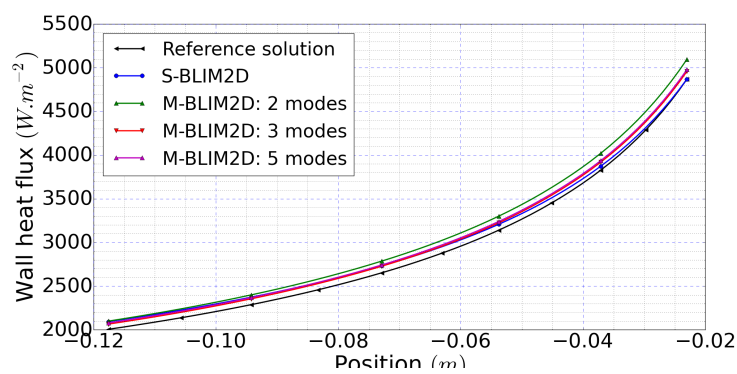

(b) Wall heat flux, zoom pressure side

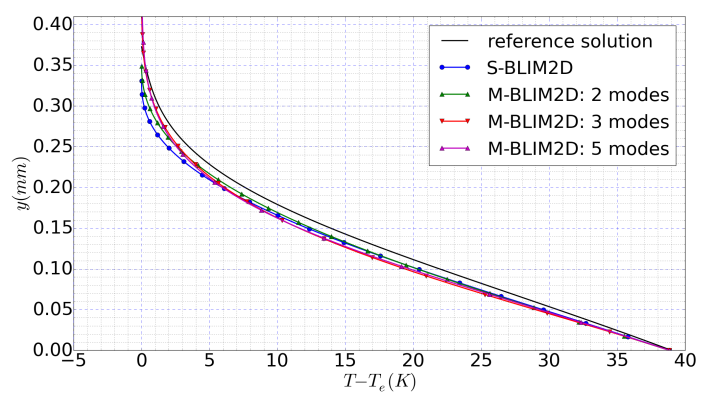

(d) Temperature profile in $x=-0.03 m$ pressure side

Fig. 8 NACA 0012 profile with a $4^{\circ}$ angle of attack and a constant wall temperature of $T_{w}=303.15 K$ 


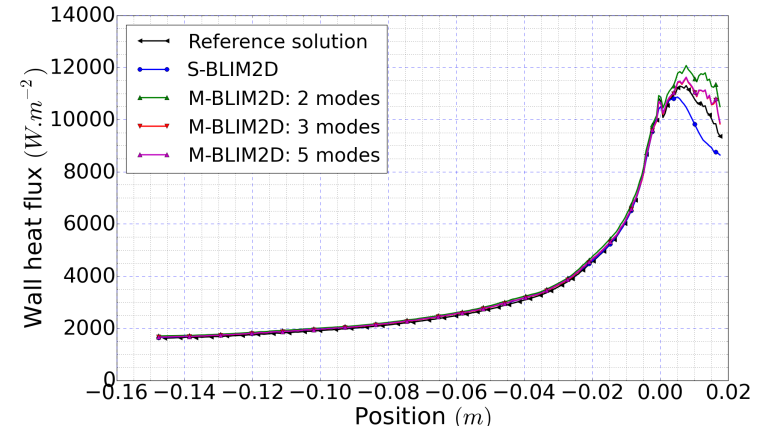

(a) Wall heat flux

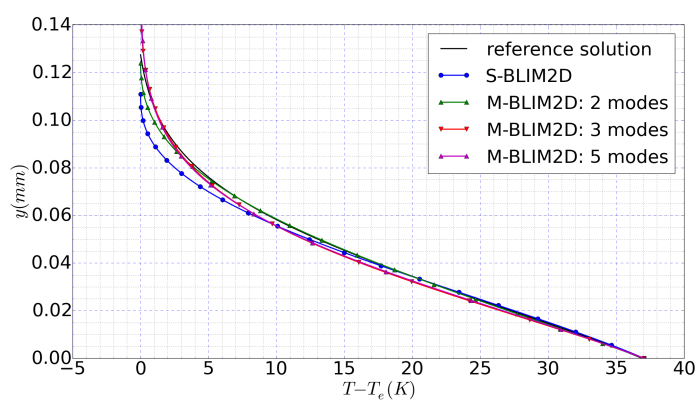

(b) Temperature profile in $x=0.01 \mathrm{~m}$, suction side

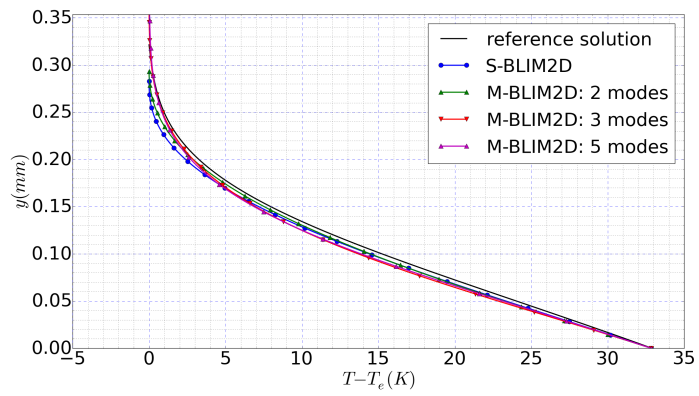

(c) Temperature profile in $x=0.02 m$ pressure side

Fig. 9 GLC 305 profile with a 4 angle of attack and a constant wall temperature of $T_{w}=303.15 \mathrm{~K}$

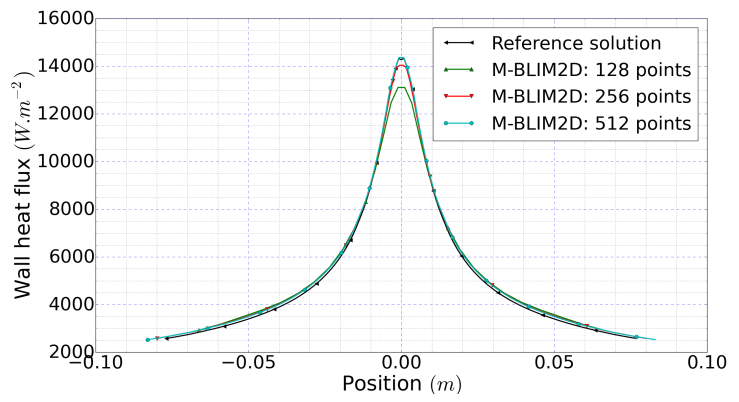

Fig. 10 Mesh convergence for the wall heat flux with a $0^{\circ}$ angle of attack and a constant wall temperature of $T_{w}=303.15 K .3$ modes are used for M-BLIM

\section{Wing profile: Non isothermal conditions}

Non uniform wall temperature on wing profiles correspond to the cases for which the method has been designed. Figure 11 refers to the same conditions as the case depicted by figure 7 (that is to say a $0^{\circ}$ AoA NACA 0012 profile with $M_{\infty}=0.25, P_{\infty}=101325 \mathrm{~Pa}, T_{\infty}=263.15 \mathrm{~K}$ ) but with a wall temperature varying linearly with the distance to the stagnation point. The wall temperature at the stagnation point is $T_{w}=323.15 \mathrm{~K}$ and the wall temperature gradient is imposed at $\left|\vec{\nabla} T_{w}\right|=-400 \mathrm{~K}_{\mathrm{w}} \mathrm{m}^{-1}$. The results are similar to the case on a wedge configuration (figure 5), the use of the modal method allowing to describe accurately the wall heat flux for an extended distance. The more modes are used, the longer it keeps being accurate. While 3 modes may seem to yield more accurate results in this instance, should a larger gradient be imposed or the laminar regime extend further, 3 modes would start to depart from the solution while 5 modes would keep up, as was shown in figure 5. The temperature profiles 11(b-c) show the same behaviour as was described in figure 5 for auto similar solutions with the same wall temperature.

Figure 12 refers to the previous conditions but with a $4^{\circ}$ angle of attack causing the maximum of heat flux to be 


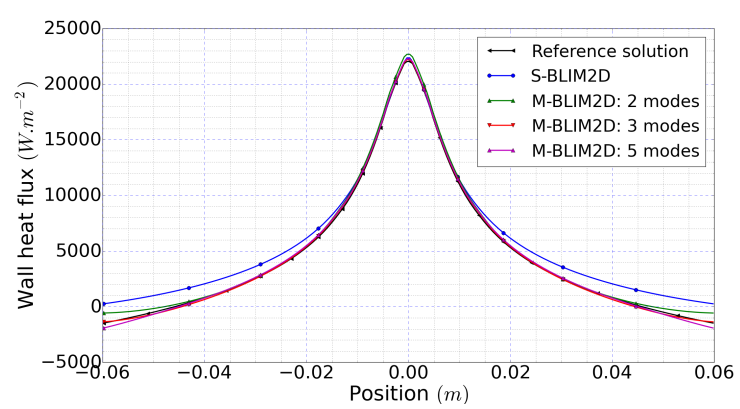

(a) Wall heat flux

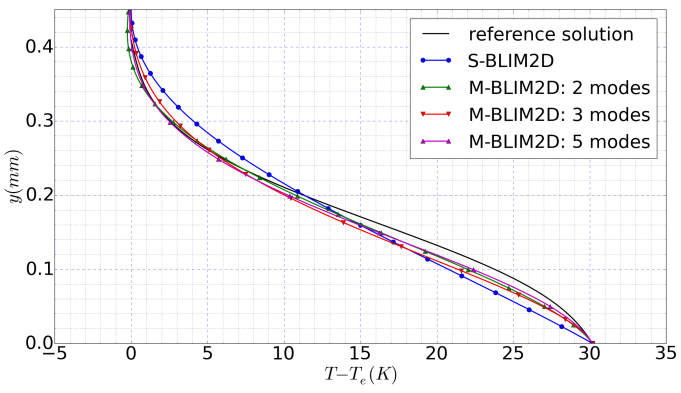

(b) Temperature profile in $x=0.04 m$

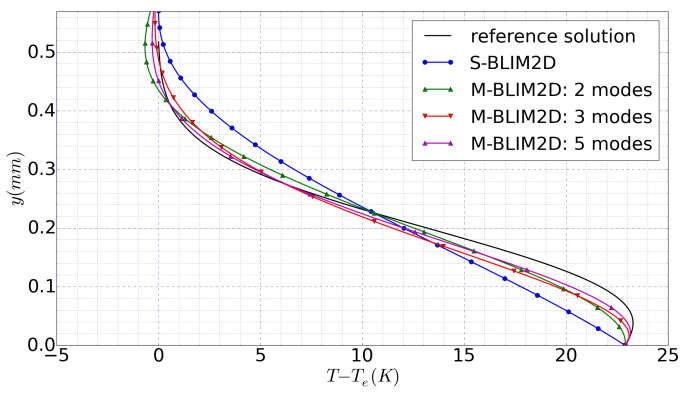

(c) Temperature profile in $x=0.05 m$

Fig. 11 NACA 0012 profile with a 0 angle of attack, an imposed wall temperature $T_{w}=323.15 \mathrm{~K}$ at stagnation point and $\nabla_{x}\left(T_{w}\right)=-400 K . m^{-1}$

reached on the suction side while the attached laminar section size is reduced on the suction side. The modal method features slight oscillations for the wall heat flux after the maximum of the wall heat flux making it less accurate than the single equation method over a short distance. On the other hand, on pressure side it shows better results than the single equation integral method over the entirety of the section.

Figure 13 refers to the same conditions as the case depicted by figure (figure $7 \mathrm{p}$ but with a wall temperature at the stagnation point of $T_{w}=288.15 \mathrm{~K}$ and a wall temperature gradient of $\left|\vec{\nabla} T_{w}\right|=+200 \mathrm{~K} . \mathrm{m}^{-1}$. The modal method with 2 modes yields worst results than the single equation method for this case. However with more modes, it yields more accurate results near the stagnation point, before departing from the solution, the error being the same as the single equation method afterwards. The temperature profile using 3 or more modes is once again more accurate at the top of the boundary layer than at the middle.

\section{Conclusion}

A thermal integral method for laminar flows, previously developed at ONERA, was presented. While it yields good results on uniform wall temperature it does not on a non uniform wall temperature. As a consequence, a second thermal integral method which is based on a modal approach was developed in order to describe more accurately such cases. Both methods were compared to a reference solution obtained from a code solving directly the Prandtl equations. The modal method yields clearly better results when the wall temperature draws closer to the external temperature the further downstream. In the opposite case, that is to say when the wall temperature becomes closer to the external temperature the further upstream, then the modal method does not show significant improvement over the previous method. While not shown in this paper, the modal method showed also better behaviour for limited wall temperature discontinuities. Both methods have been only implemented for 2 dimension problems but the extension to three dimensions is straightforward for both. The modal method is strictly explicit but the source terms will be implicited in order to increase the method robustness as well as to reduce calculation time. The effect of compressibility in the thermal boundary layer will also be taken into account for the modal method. Both methods will be incorporated in the ONERA icing suit, IGLOO2D in order to asses their behaviour in their intended use on real icing cases. In the long run, the modal method should be 


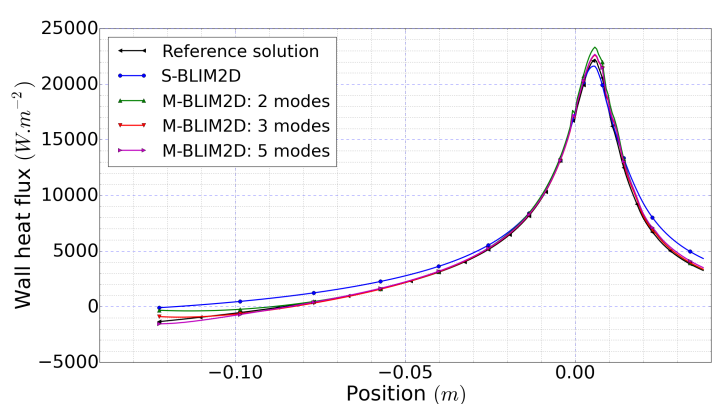

(a) Wall heat flux

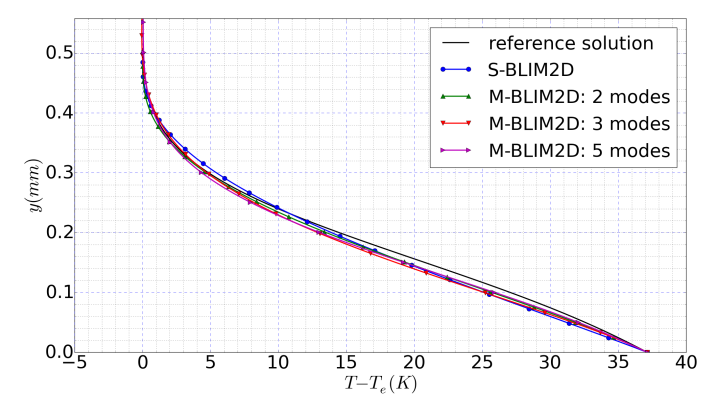

(c) Temperature profile in $x=-0.05 m$ on pressure side

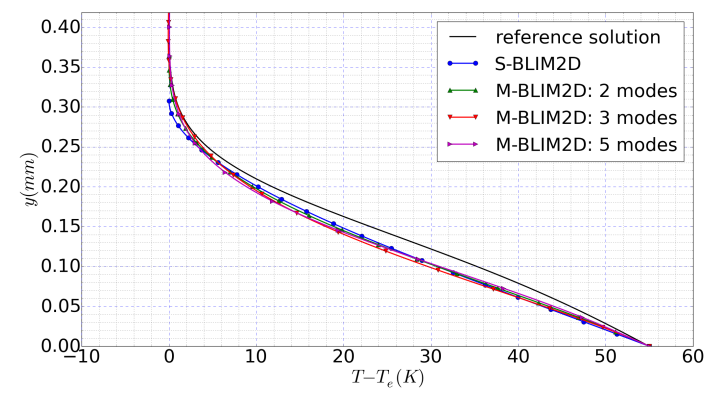

(b) Temperature profile in $x=0.03 m$ on the suction side

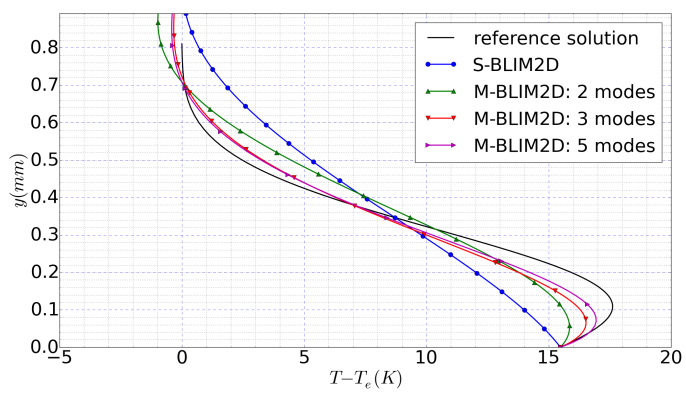

(d) Temperature profile in $x=0.1 \mathrm{~m}$ on pressure side

Fig. 12 NACA 0012 profile with a $4^{\circ}$ angle of attack, an imposed wall temperature $T_{w}=323.15 \mathrm{~K}$ at stagnation point and $\nabla_{x}\left(T_{w}\right)=-400 K . m^{-1}$

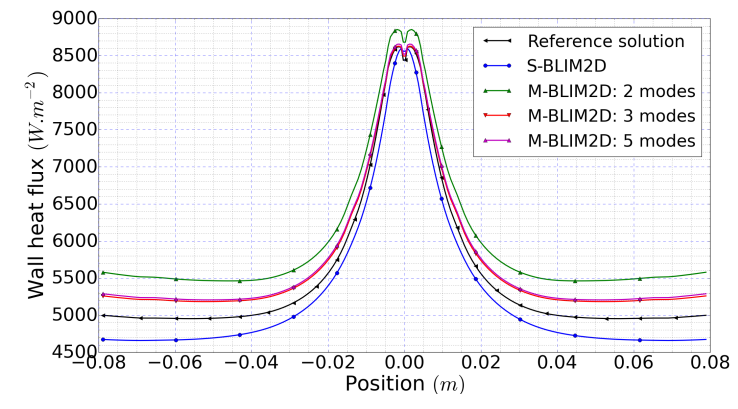

(a) Wall heat flux

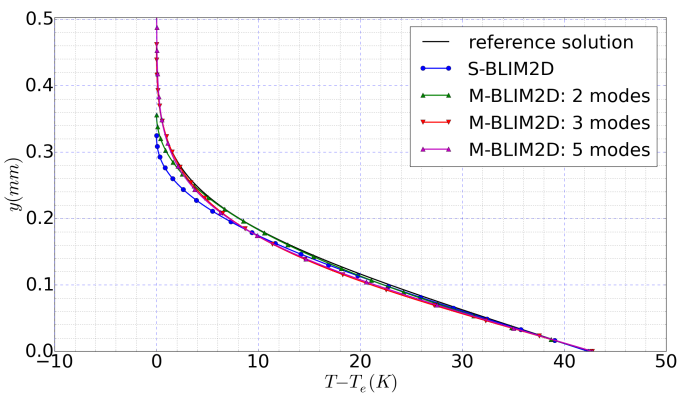

(b) Temperature profile in $x=0.02 m$

Fig. 13 NACA 0012 profile with a $0^{\circ}$ angle of attack, an imposed wall temperature $T_{w}=288.15 K$ at stagnation point and $\nabla_{x}\left(T_{w}\right)=+200 K . m^{-1}$

investigated in turbulent flows. This should require some work to adapt the sources term at least, if not the projection basis as well.

\section{References}

[1] Bayeux, C., Radenac, E., and Villedieu, P., "Theory and Validation of a 2D Finite-Volume Integral Boundary-Layer Method for Icing Applications.” AIAA Journal, Vol. 57, No. 3, 2019, pp. 1092-1112. https://doi.org/10.2514/1.J057461

[2] Bayeux, C., "Méthode Intégrale pour la couche limite tridimensionnelle. Applications au givrage," Ph.D. thesis, Institut Superieur de l'Aeronautique et de l'Espace (ISAE), 2017.

[3] Radenac, E., Bayeux, C., and Villedieu, P., "Use of a Two-Dimensional Finite Volume Integral Boundary-Layer Method for IceAccretion Calculations.” AIAA Journal, Vol. 58, No. 4, 2020, pp. 1592-1606. https://doi.org/https://doi.org/10.2514/1.J058701 
[4] Radenac, E., Kontogiannis, A., Bayeux, C., and Villedieu, P., "An extended rough-wall model for an integral boundary layer model intended for ice accretion calculations," 10th AIAA Atmospheric and Space Environments Conference - AVIATION 2018 ATLANTA, USA, AIAA 2018-2858, 2018. https://doi.org/10.2514/6.2018-2858.

[5] J.Cousteix, Aérodynamique et Couche limite laminaire, Cepadues, 1989.

[6] Kays, W., and Crawford, M., Convective heat and mass transfer, McGraw-Hill, 1993.

[7] B.Aupoix, "Couches Limites Bidimensionnelles Compressibles. Descriptif et mode d'emploi du code CLICET - Version 2015," Tech. rep., ONERA, 2015. 